\title{
Adenocarcinoma primário multicêntrico com 12 focos. Relato de caso e revisão da literatura.
}

\author{
Multicenter primary adenocarcinoma with 12 foci: case report and literature review
}

\author{
SHEILA JORGE ADAD ${ }^{1}$, CARLOS AUGUSTO CANTERAS RAPOSO CAMARAํ, JULIANA MOTA PEREIRA ${ }^{1}$, JOSÉ \\ DO CARMO JR ${ }^{1}$, ADILHA MISSON RUA MICHELETTI ${ }^{1}$.
}
${ }^{1}$ Disciplina de Patologia Especial da Universidade Federal do Triângulo Mineiro (UFTM) - Uberaba (MG), Brasil. Trabalho realizado na Disciplina de Patologia Especial da Universidade Federal do Triângulo Mineiro,
Uberaba (MG), Brasil.

\begin{abstract}
ADAD SJ, CAMARA CACR, PEREIRA JM, CARMO JR J, MICHELETTI AMR. Adenocarcinoma primário multicêntrico com 12 focos. Relato de caso e revisão da literatura. Rev bras Coloproct, 2011;31(2): 205-209.

RESUMO: Relata-se caso raro de adenocarcinoma primário multicêntrico sincrônico em intestino delgado, apêndice cecal e intestino grosso, em homem de 82 anos, com quadro de abdome agudo inflamatório. Foi submetido à laparotomia exploradora, observando-se lesão intestinal estenosante e infiltrativa no ângulo hepático e múltiplas aderências entre as alças do intestino delgado. Foi realizada hemicolectomia direita. $O$ estudo anatomopatológico mostrou 12 focos de adenocarcinomas primários comprometendo intestino delgado (oito focos), válvula ileocecal, apêndice cecal e intestino grosso (cólon ascendente e transverso).
\end{abstract}

Palavras-chave: adenocarcinoma;intestinos delgado e grosso; apêndice cecal; íleo; câncer de cólon.

\section{INTRODUÇÃO}

Adenocarcinoma multicêntrico comprometendo o intestino delgado e grosso é bastante raro ${ }^{1}$. Para ser considerado câncer primário múltiplo, as lesões devem ser malignas, independentes e não resultarem de metástases. Para serem denominadas de sincrônicas, as lesões devem ser diagnosticadas simultaneamente ou dentro de um intervalo de até seis meses ${ }^{1}$. Para serem considerados como primários é necessário que se identifique microscopicamente a transição do epitélio normal para o maligno na borda do tumor ${ }^{2}$. Encontramos na literatura, através da pesquisa no PubMed, apenas nove casos de adenocarcinomas sincrônicos multicêntricos de intestino delgado e grosso ${ }^{1,3-10}$, excluindo os adenocarcinomas associados às síndromes hereditárias de câncer colo-retal, como a polipose adenomatosa fa- miliar, nas quais os carcinomas do intestino delgado são em geral em torno da ampola de Vater ${ }^{11}$.

\section{RELATO DO CASO}

Paciente de 82 anos, do sexo masculino, branco, encaminhado ao serviço de cirurgia geral da unidade de emergência, com queixa de dor abdominal intensa e difusa há três dias. Referia obstipação intestinal há seis meses (até quatro dias sem evacuar), tendo apresentado dois episódios de enterorragia. Queixavase, ainda, de anorexia, com emagrecimento de $10 \mathrm{~kg}$ neste período e referia ter diabete melito tipo II, controlado com medicação oral e dieta. Três meses antes esteve internado, nesse mesmo hospital, com quadro semelhante de suboclusão intestinal. Nessa internação anterior, foram feitas duas tentativas de colonoscopia,

Fonte de financiamento: não há

Conflito de interesse: nada a declarar

Recebido em: 08/06/2010

Aprovado em: 09/07/2010 
que foram insatisfatórias, pois não se conseguiu uma boa limpeza do intestino; na terceira tentativa, a mucosa parecia normal e não havia fecaloma. $\mathrm{O}$ enema opaco feito nessa internação anterior evidenciou apenas alongamento do sigmoide.

O paciente apresentava estado geral regular, hipocorado $(2+/ 4+)$, abdome globoso, distendido, timpânico difusamente, doloroso à palpação superficial e profunda, descompressão brusca negativa e ruídos hidroaéreos diminuídos. Relatava ter apresentado, no mesmo dia, episódio de eliminação de fezes e flatos. Os exames solicitados à admissão mostraram leucocitose com desvio à esquerda e série vermelha normal; glicemia, ureia, creatinina, amilase e eletrólitos sem alterações significativas. Ultrassonografia de abdome revelou grande quantidade de líquido intraabdominal, sendo anecoide em espaço de Morrison e esplenorrenal, heterogêneo e espessado em fundo de saco posterior, com alças do reto-sigmoide distendidas e com paresia. O paciente foi submetido à laparotomia mediana ampla, observando-se peritonite difusa com grande quantidade de secreção fibrinopurulenta na cavidade abdominal e massa que aderia o intestino delgado ao ceco e apêndice cecal. Após serem desfeitas as aderências e inspecionadas as alças do intestino grosso, notou-se lesão estenosante próxima ao ângulo hepático e nódulos confluentes endurecidos e esbranquiçados no tecido adiposo em torno do cólon transverso distal. Realizou-se hemicolectomia direita com ileostomia terminal. O paciente evoluiu bem no pós-operatório imediato e recebeu alta hospitalar no sexto dia pós-operatório; porém, retornou à unidade após dez dias com quadro de astenia, febre e dor abdominal, identificando-se à tomografia de abdome, abscesso no flanco direito. Apesar da conduta adotada, o paciente evoluiu para septicemia refratária e faleceu 30 dias após a cirurgia.

\section{Exame anatomopatológico}

O exame macroscópico mostrou peça cirúrgica constituída por íleo terminal, apêndice cecal, ceco, cólon ascendente e parte do cólon transverso em monobloco. O segmento de íleo distal media 80 $\mathrm{cm}$ e o segmento de intestino grosso, $60 \mathrm{~cm}$. Havia focos de exsudado fibrinopurulento, especialmente em torno da válvula ileocecal e múltiplas aderências entre as alças do intestino delgado. Em 12 locais, o lúmen estava discretamente reduzido e a parede espessada, com ulcerações na mucosa que mediam entre 0,5 e $0,8 \mathrm{~cm}$, oito localizadas no intestino delgado (Figura 1A), uma na válvula ileocecal, infiltração do apêndice cecal (Figura 2A) e dois focos no intestino grosso localizados no cólon ascendente (Figura 3A) e transverso, distando o último $1 \mathrm{~cm}$ da margem distal de ressecção. $\mathrm{O}$ apêndice cecal apresentava-se endurecido e reduzido de volume, medindo $3 \times 1,5 \times 1 \mathrm{~cm}$, também com aspecto de infiltração tumoral. A superfície de corte dessas ulcerações e retrações mostrava delgada orla de te-
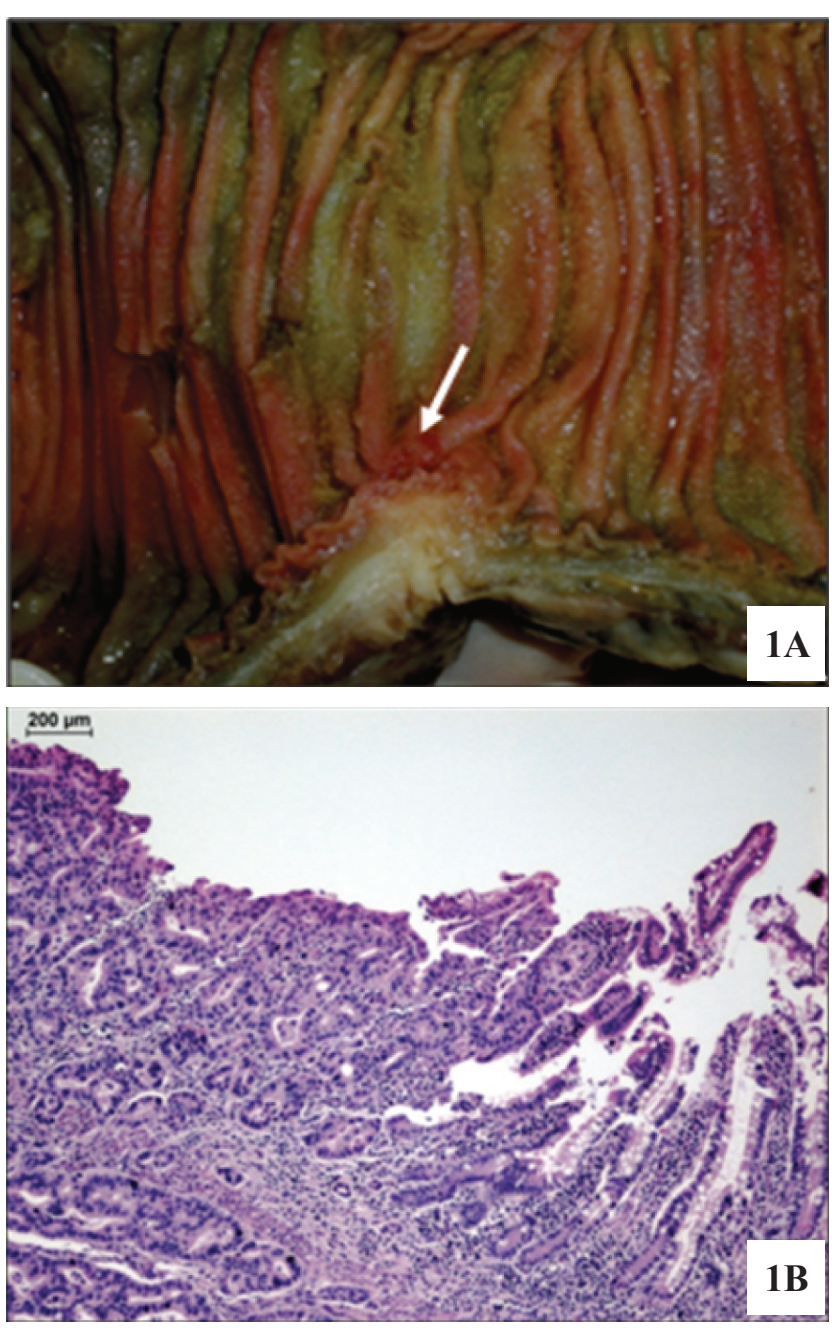

Figura 1. (A) Peça cirúrgica mostrando tumor ulcerado invadindo todas as camadas no íleo (seta). (B) Fotomicrografia do íleo representando o adenocarcinoma (parte inferior direita), adjacente à área de mucosa não neoplásica (HematoxilinaEosina $-H E-100 \times)$. 

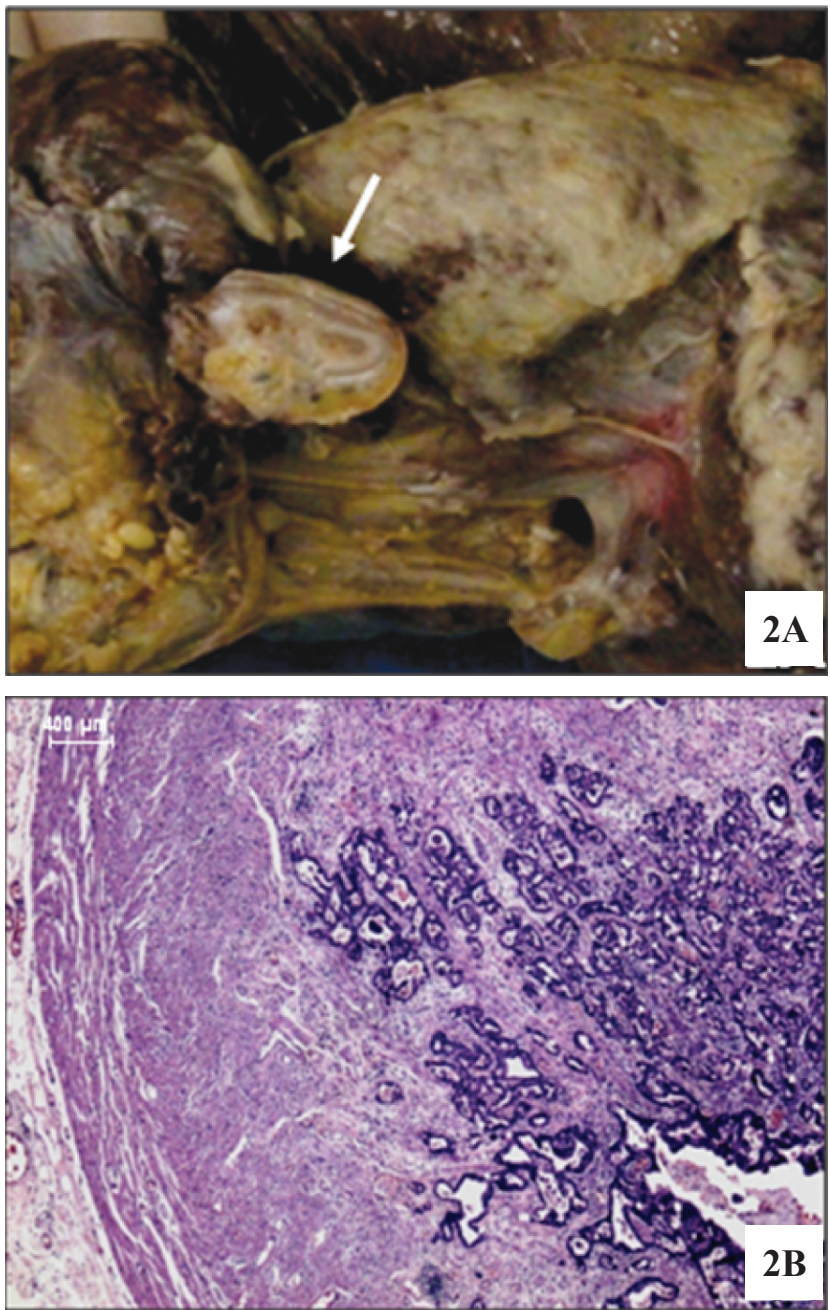

Figura 2 (A) Apêndice cecal (seta) com mucosa e submucosa substituídas pela neoplasia. Observar peritonite fibrinopurulenta, em torno, devido à perfuração do tumor na região ileocecal. (B) Aspecto microscópico do adenocarcinoma no apêndice $(H E-100 \times)$.

cido de tonalidade creme, com tecido brancacento subjacente. Havia perfuração na válvula ileocecal. $\mathrm{Na}$ mucosa do intestino grosso observavam-se, ainda, dois pequenos pólipos pediculados medindo 0,5 e $0,8 \mathrm{~cm}$, distando no mínimo $10 \mathrm{~cm}$ da margem de ressecção distal. Foram vistos no mesocólon transverso nódulos confluentes medindo $3,5 \times 2 \mathrm{~cm}$ sugestivos de metástases em linfonodos.

O exame microscópico evidenciou 12 focos de adenocarcinoma ulcerado, bem ou moderadamente diferenciado no intestino delgado (Figura 1B), válvula ileocecal, apêndice cecal (Figura 2B) e intestino
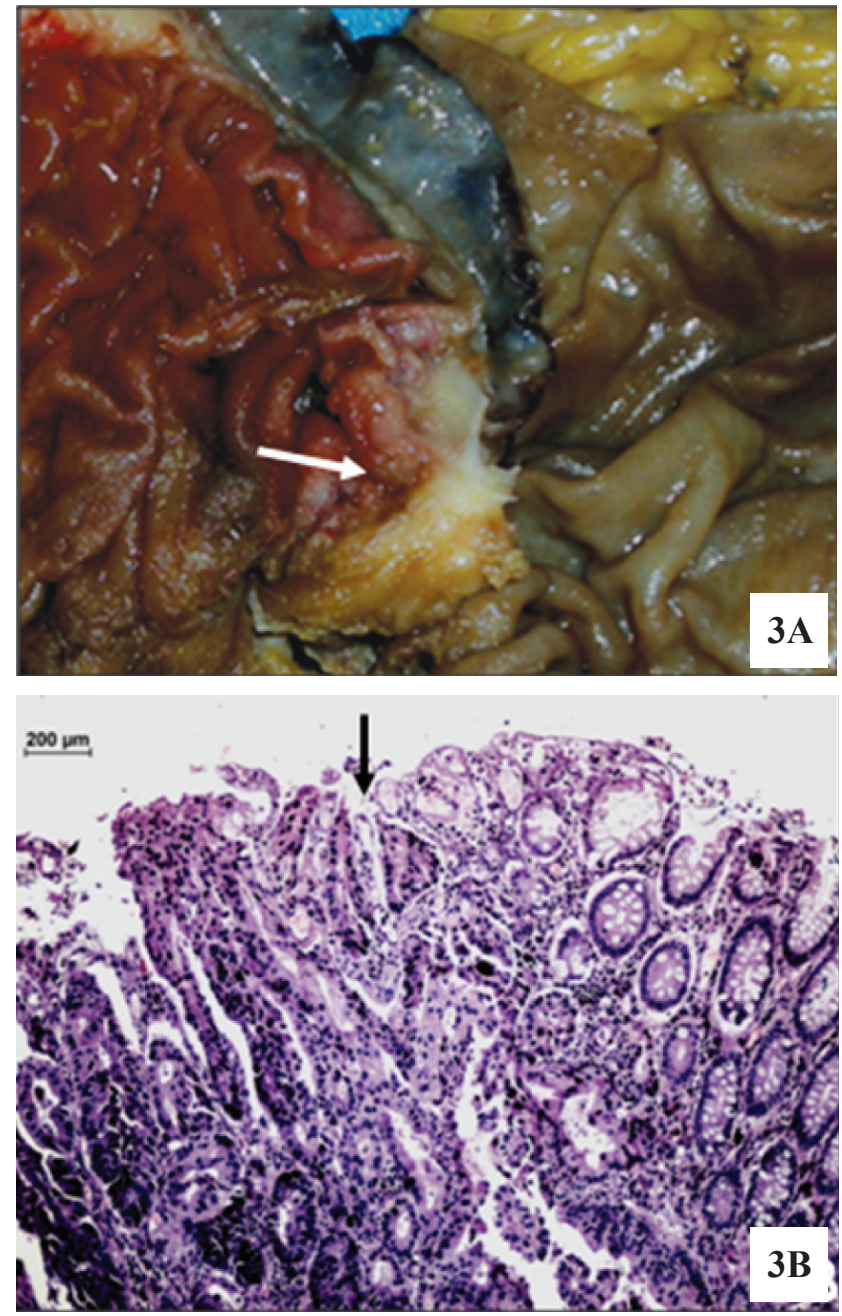

Figura 3. (A)Mucosa do intestino grosso com foco de adenocarcinoma ulcerado no cólon ascendente (seta). (B) Detalhe microscópico da Figura 3A, mostrando adenocarcinoma bem diferenciado à esquerda e mucosa normal à direita da seta (HE - 200x).

grosso (cólon ascendente - Figura 3B - e transverso), frequentemente invadindo todas as camadas e perfurando a válvula ileocecal (estadiamento pT4); peritonite fibrinopurulenta em organização; metástases nodulares no tecido adiposo em torno do cólon transverso (provável pN2), não sendo possível determinar se tratavam-se ou não de linfonodos totalmente substituídos pela neoplasia. Os linfonodos da região mesentérica não apresentavam neoplasia. Havia ainda dois pequenos adenomas tubulares, com displasia de baixo grau no cólon ascendente e transverso. Os múltiplos focos de adenocarcinoma foram con- 
siderados como primários porque tinham ulceração central e apresentavam, na transição para o tecido normal, áreas de displasia e carcinoma in situ. Entre as lesões, a mucosa era normal. As lesões tinham tamanhos semelhantes, ou seja, sem uma lesão predominante. Como o aspecto macroscópico das lesões do intestino delgado lembrava carcinoide, foi feito estudo imunohistoquímico, que mostrou positividade para citoceratina 20 (CK 20) e antígeno cárcinoembriônico (CEA), e negatividade para sinaptofisina e cromogranina, confirmando o diagnóstico de adenocarcinoma.

\section{DISCUSSÃO}

Adenocarcinomas multifocais primários comprometendo intestinos delgado e grosso, sincronicamente, são bastante raros. Encontramos, revisando a literatura (PubMed), apenas nove relatos ${ }^{1,3-10}$, excluindo os adenocarcinomas associados às síndromes hereditárias de câncer colorretal, como a polipose adenomatosa familiar (Tabela 1). Até mesmo nessas síndromes não são comuns adenocarcinomas de intestino delgado sincrônicos com os de intestino grosso e, em geral, quando ocorrem localizam-se em torno da ampola de Vater ${ }^{11}$. Nesse levantamento, encontramos apenas um caso de doença de Crohn com neoplasia sincrônica no intestino delgado e grosso ${ }^{3}$, assim como somente um caso semelhante associado à irradiação pélvica 5 . Os demais casos, assim como o nosso, parecem não ter fatores predisponentes óbvios associados.
Em um estudo retrospectivo, compreendendo um período de 31 anos, Ouriel et al. ${ }^{2}$ encontraram 65 casos de adenocarcinoma de intestino delgado (excluindo os carcinomas da ampola de Vater), $15 \%$ dos quais estavam localizados no íleo. Aqueles autores descrevem um único caso com dois focos sincrônicos de adenocarcinoma no intestino delgado, localizados no jejuno e íleo, em áreas comprometidas pela doença de Crohn. Relataram, ainda, que outros 16 pacientes, com foco único de adenocarcinoma no intestino delgado, apresentavam associação com outras neoplasias malignas, sendo adenocarcinoma do cólon em 12 casos; porém, não referem serem os tumores sincrônicos.

Dentre os nove casos que encontramos na literatura ${ }^{1,3-10}$, cinco apresentavam apenas dois focos de adenocarcinoma (Tabela 1), dois casos tinham três focos e, apenas dois casos, apresentavam mais de dez focos sincrônicos. O caso ora relatado é o terceiro com mais de dez focos; entretanto, difere dos demais com numerosos focos, por apresentar comprometimento também do apêndice cecal, além do cólon. Acometimento sincrônico do apêndice cecal foi visto apenas em um caso anteriormente, no qual o comprometimento do intestino grosso foi apenas do apêndice ${ }^{10}$. Outro aspecto diferente, em nosso caso, foi o acometimento da válvula ileocecal, não encontrado em nenhum dos já relatados e que levou à perfuração, gerando um quadro de abdome agudo inflamatório.

O diagnóstico de adenocarcinoma no íleo em geral é tardio. As manifestações mais frequentes são dor abdominal, obstrução, sangramento e anemia ${ }^{6}$. Entre-

Tabela 1. Revisão de casos relatados de adenocarcinomas primários multicêntricos sincrônicos de intestino delgado e grosso.

\begin{tabular}{lcccc}
\hline Primeiro autor & Ano & $\mathbf{N}^{\mathbf{0}}$ de lesões & Localização no intestino delgado & Localização no intestino grosso \\
\hline Wagner $^{10}$ & 1982 & 13 & Íleo & Apêndice \\
Reddy $^{*}$ & 1990 & 2 & Jejuno & Ascendente \\
Liu $^{1}$ & 1996 & $>30$ & Duodeno, jejuno, íleo & Ascendente, tranverso \\
Lee $^{7}$ & 1997 & 3 & Jejuno & Ceco \\
Despande $^{4}$ & 1998 & 2 & Jejuno & Ascendente \\
Gajraj $^{5 * *}$ & 1998 & 2 & Íleo & Reto \\
Baisse $^{3 * * *}$ & 2004 & 2 & Íleo & Ceco \\
Hosono $^{6}$ & 2006 & 2 & Íleo & Transverso \\
Leong $^{8}$ & 2008 & 3 & Jejuno & Ceco, tranverso \\
Adad & 2009 & 12 & Íleo, válvula ileocecal & Apêndice ascendente, transverso \\
\hline
\end{tabular}

*Adenocarcinoma de cólon transverso dois anos antes; **irradiação pélvica 20 anos antes; ***doença de Crohn. 
tanto, essa região do intestino delgado é a mais difícil do trato gastrointestinal para ser examinada, tanto por endoscopia, quanto por métodos radiológicos convencionais e, por isso, o diagnóstico frequentemente é feito em fase avançada da doença. Observamos, no presente caso, falha na detecção através da colonoscopia, em parte devido à dificuldade em se obter uma boa limpeza do intestino, em parte provavelmente porque as lesões eram pequenas e pouco evidentes, lembrando mais pequenas retrações e erosões, macroscopicamente, do que neoplasia, mesmo seis meses após o início dos sintomas e três meses depois da realização da colonoscopia e do enema opaco. Falha simi- lar ocorreu no caso descrito por Hosono et al. ${ }^{6}$, que só conseguiram fazer o diagnóstico após a utilização de cápsula endoscópica. A maioria dos outros casos citados também apresentou história pouco específica, sinais e sintomas vagos, retardando o diagnóstico, que em vários casos só foi feito através do ato cirúrgico e em estágio avançado da doença, quando o prognóstico em geral é muito ruim ${ }^{6}$.

Em conclusão, relatamos caso raro de adenocarcinoma primário multicêntrico no intestino delgado e grosso, que através da pesquisa da literatura pelo PubMed parece ser o terceiro caso com mais de dez focos sincrônicos.

ABSTRACT: A rare case of synchronous multicenter primary adenocarcinoma in the small intestine, cecal appendix and large intestine, in an 82-year-old man with a condition of acute abdominal inflammation, is reported. He underwent exploratory laparotomy, and a stenosing and infiltrative intestinal lesion was seen in the hepatic angle, along with multiple adherences between the loops of the small intestine. Right hemicolectomy was performed. The anatomopathological evaluation showed 12 foci of primary adenocarcinomas affecting the small intestine (eight foci), ileocecal valve, cecal appendix and large intestine (ascending and transverse colon).

Keywords: adenocarcinoma; small and large intestines; cecal appendix; ileum; colonic neoplasm.

\section{REFERÊNCIAS}

1. Liu KJM, Nadimpalli V, Atten MJ, Chang HR, Finkelstein $\mathrm{SD}$, Mak K, et al. Multicentric primary adenocarcinomas of the midgut: the first case report. Am J Gastroenterol 1996;91:1239-43.

2. Ouriel K, Adams JT. Adenocarcinoma of the small intestine. Am J Surg 1984;147:66-71.

3. Baisse B, Fontolliet C, Bian YS, Vuilleumier H, Benhattar J. Synchronous ileal and colonic adenocarcinomas associated with Crohn's disease: report of a case with a focus on genetic alterations and carcinogenesis. J Clin Pathol 2004;57:885-7.

4. Despande AA, Thapar VK, Bakshi GD, Joshi A, Rao PP, Supe AN. Synchronous primary adenocarcinoma of small and large bowel. Indian J Gastroenterol 1998;17:156.

5. Gajraj H, Davies DR, Jackson BT. Synchronous small and large bowel cancer developing after pelvic irradiation. Gut 1998;29:126-8.

6. Hosono S, Ohira M, Maeda K, Muguruma K, Nishihara T, Inoue $\mathrm{T}$, et al. Synchronous adenocarcinomas of the ileum and transverse detected by capsule endoscopy: Report of a case. Surg Today 2006;36:663-5.

7. Lee J, Tamhane R, Parks TG. Caecal adenocarcinoma with multiple synchronous small intestinal adenocarcinoma.
Postgrad Med J 1997;73:729-31.

8. Leong BDK, Ramu P, Kumar VM, Chuah JA. Synchronous adenocarcinoma of caecum, transverse colon and jejunum. Med J Malaysia 2008;63:148-9.

9. Reddy VB, Husain AN, Abraham K, Castelli MJ, Gattuso P. Synchronous adenocarcinomas of jejunum and cecum following transverse colon carcinoma. A case study. Mt Sinai J Med 1990;57:34-6.

10. Wagner KM, Thompson J, Herlinger H, Caroline D. Thirteen primary adenocarcinomas of the ileum and appendix. Cancer 1982;49:797-801.

11. Akatsu T, Aiura K, Takahashi S, Kameyama K, Kitajima M, Kitagawa Y. Recurrent pancreatitis caused by ampullary carcinoma and minor papilla adenoma in familial polyposis: report of a case. Surg Today 2008;38:440-4.

\section{Endereço para correspondência:}

Sheila J. Adad.

Disciplina de Patologia Especial

Universidade Federal do Triângulo Mineiro.

Av. Getúlio Guaritá, 130 - CEP: 38.025-440 - Uberaba (MG), Brasil.

Tel (34) 3318-5152 - Fax (34) 3313-7105

E-mail: sheila.adad@gmail.com 